\title{
Membrane property and biofunction of phospholiposome incorporated with anomeric galactolipids
}

\author{
Danyang Liu', Junqi Zhang ${ }^{2 *}$, Shouhong $\mathrm{Xu}^{1 *}$ and Honglai Liu ${ }^{1}$
}

\author{
*Correspondence: \\ junqizhang@fudan.edu.cn: \\ xushouhong@ecust.edu.cn \\ ${ }^{1}$ Key Laboratory \\ for Advanced Materials \\ and Institute of Fine \\ Chemicals, School \\ of Chemistry and Molecular \\ Engineering, East China \\ University of Science \\ and Technology, 130 Meilong \\ Rd, Shanghai 200237, \\ People's Republic of China \\ ${ }^{2}$ Key Laboratory of Medical \\ Molecular Virology (Ministry \\ of Health and Ministry \\ of Education), School of Basic \\ Medical Sciences, Fudan \\ University, 138 Yixueyuan Rd, \\ Shanghai 200032, People's \\ Republic of China \\ Full list of author information \\ is available at the end of the \\ article
}

\begin{abstract}
There has been increasing interest in the construction of liposomes containing a targeting reagent for target-specific drug delivery. Glycoconjugates that can be recognized by transmembrane glycoprotein receptors have been extensively used to form glyco-liposomal drug carriers. However, the impact of anomerism, which is a common identity of natural glycoconjugates, on the glyco-liposomal properties has been hardly probed in previous studies. Here we investigate the liposomal properties of phospholipid incorporated with a pair of anomeric galactolipids. The anomeric galactoliposomes are characterized and their membrane fluidity, thermo-stability, DNA condensation efficiency and fluorescence leakage are comparatively tested. The in vitro cellular internalization effect of the galacto-liposomes is also demonstrated. This study suggests that anomerism might give distinct impact on the membrane properties and even biofunctions of glyco-liposomes.
\end{abstract}

Keywords: Glycolipid, Hybrid liposome, Interfacial behaviour, DNA condensation

\section{Background}

Liposome has the similar structure to biomembrane, such as cell membrane, and is always used as substitute in research of biomembrane. It is easy to believe that the properties of membranes should give large influence on their biofunctions. For example, lower thermal stability might increase membrane permeability and release drug quickly (Yang et al. 2014). Positive charged liposomal surface might promote interaction with cells (Rädler et al. 1997). And even, the membrane fusion should be determined partly by properties of bilayer membranes.

In the drug or DNA delivery system, liposomes can effectively protect cargoes from being biodegraded in vivo before reaching a target tissue. However, the drug delivering efficiency of commercial liposomes is usually compromised due to the lack of a 'warhead' that effectively directs them to the target. In recent years, construction of glycolipid (a saccharide covalently linked to a lipid)-incorporated liposomes (Jayaraman et al. 2013; Ueno et al. 2007; Róg et al. 2007; Ramezani et al. 2009; Stimac et al. 2012; Yin et al. 2013) has been of increasing interest since the glycosyl group of which can be specifically recognized by a transmembrane glycoprotein receptor of a target tissue (Weis et al. 1998; Marth and Grewal 2008). The recognition may then facilitate the endocytosis and/or

(c) 2016 The Author(s). This article is distributed under the terms of the Creative Commons Attribution 4.0 International License (http://creativecommons.org/licenses/by/4.0/), which permits unrestricted use, distribution, and reproduction in any medium, provided you give appropriate credit to the original author(s) and the source, provide a link to the Creative Commons license, and indicate if changes were made. 
fusion of the liposomes by the host cells, releasing an encapsulated therapeutic agent. Among them, galactoliposomes that can be recognized by the asialoglycoprotein receptors (ASGPR) expressed by the hepatocyte (Rigopoulou et al. 2012) are the most extensively developed, and are considered to be promising non-viral vectors for target-specific treatment of hepatic diseases (Sliedregt et al. 1999; Zhao et al. 2011; Symens et al. 2012; $\mathrm{Hu}$ et al. 2013; Jain et al. 2012). Monosaccharides are linked to each other or with an aglycon in either the $\alpha$ - or $\beta$-configuration (at the anomeric carbon) to form cell-surface 'antennas', such as glycoproteins or glycolipids, that transmit signals between cells (Varki 1993; Bertozzi and Kiessling 2001; Seeberger 2005; Hart and Copeland 2010). These anomeric counterparts may, however, possess different physiochemical attributes, thereby leading to a diversification of biological function. In addition, some synthetic anomeric glycolipids have shown distinct pharmacological properties (He et al. 2011). Despite the fruitful results obtained in the investigation of glycolipid-based liposomal transfection (Ueno et al. 2007), to our knowledge, investigations on the properties of anomeric galacto-liposomes have been elusive.

We had studied and reported on interfacial behaviors of anomeric galactolipid in DPPE monolayer prepared by LB technique. The interaction between molecules and molecular arranging manner were found to be depended on the ratios of galactolipid (Song et al. 2012). Here we use a pair of $\alpha$-(1) and $\beta$-galacolipid(2) (Fig. 1) to investigate comparatively their liposomal properties of a DPPE-based liposome (Fig. 2). The dependencies and relationships between their thermo-stability/membrane fluidity/membrane density and the biofunctions, such as drug leakage efficiency and DNA condensation efficiency have been investigated. What kind of membrane might benefit the drug or DNA delivery has been discussed.

\section{Methods}

\section{Preparation of liposome}

$5.0 \mathrm{mg}$ of DPPE (99 \%, Lipoid, Germany) and $1.0 \mathrm{mg}$ of cationic cholesterol (Sigma, USA) (molar ratio $\approx 3 / 1$ ) were dissolved in $5 \mathrm{~mL}$ of chloroform, and $2.5 \mathrm{mg}$ of galactolipid

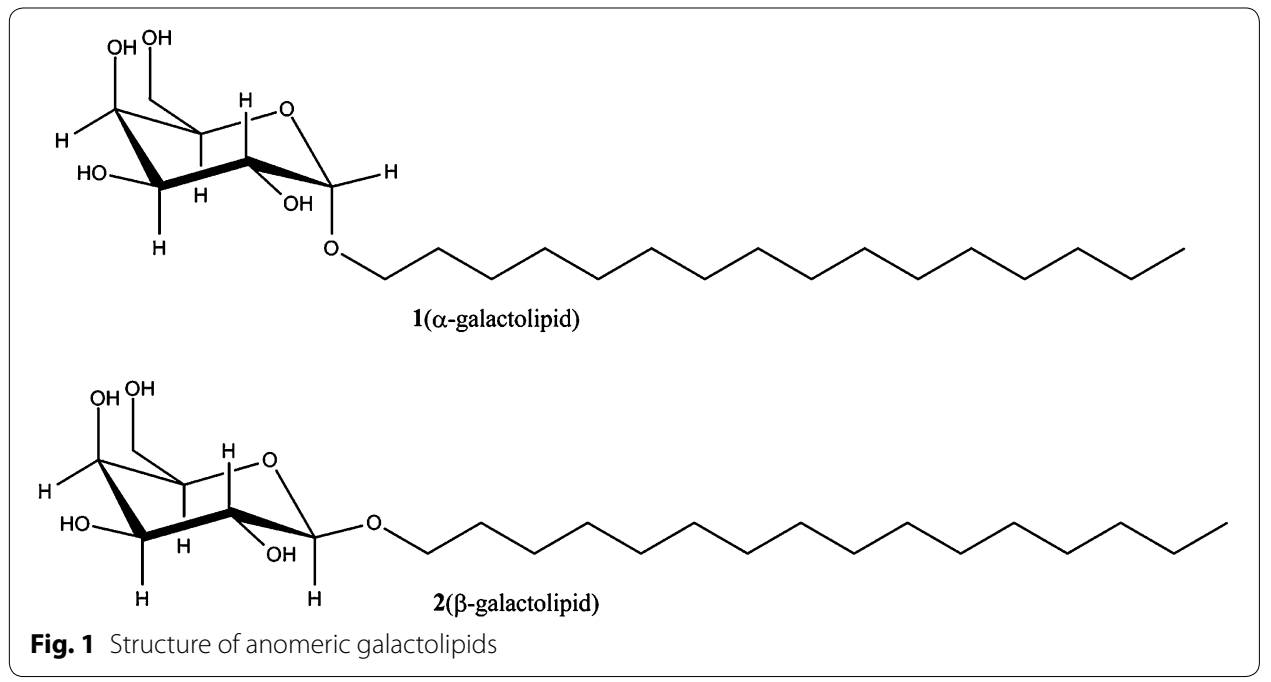




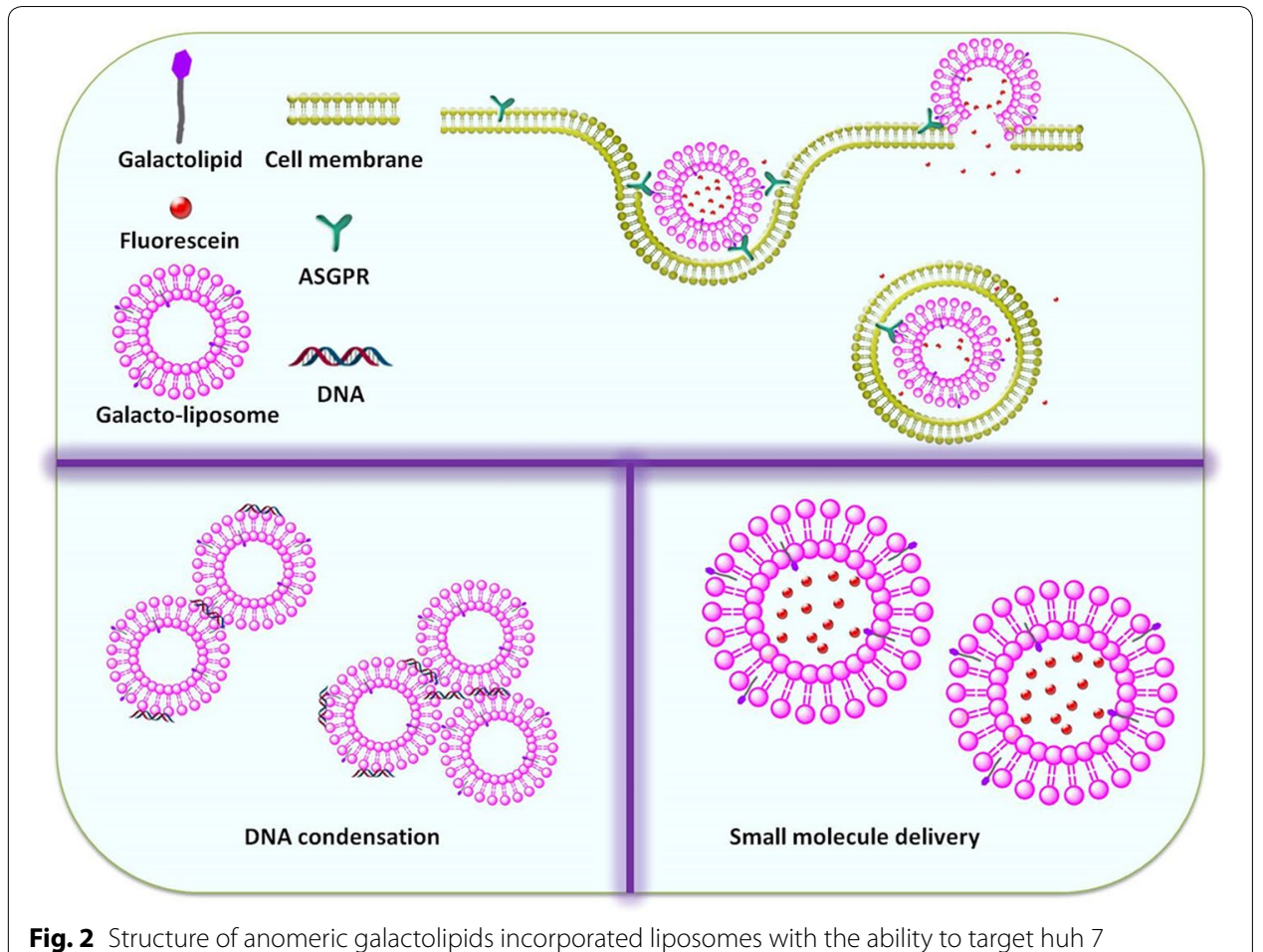

was dissolved in $5 \mathrm{~mL}$ of dichloromethane containing minute quantity of methyl alcohol. Then the two solutions were mixed with different molar ratios. The organic solvents were removed by a rotary evaporator for $45 \mathrm{~min}$ at $37^{\circ} \mathrm{C}$ and another $1 \mathrm{~h}$ under vacuum at room temperature. The lipid film was hydrated with ultrapure water (for characterization experiments) or Tris- $\mathrm{HCl}$ buffer solution (10 mM, pH 7.4, for biology experiments), and the suspension was sonicated in a bath sonicator under $65{ }^{\circ} \mathrm{C}$ for $30 \mathrm{~min}$. The suspension was then extruded (11 times) through a polycarbonate membrane with a pore diameter of $200 \mathrm{~nm}$ (Whatman, UK) by using LiposoFast (LF-1, Avestin, Canada). The particle sizes were about $150 \pm 10 \mathrm{~nm}$ measured by a dynamic light scattering (Zetasizer Nano S, Malvern, UK). Their phase transition temperatures were determined on a differential scanning calorimetry DSC (Setaram DSC III, USA) at a scan rate of $1{ }^{\circ} \mathrm{C} / \mathrm{min}$ covering a temperature range of $25-70{ }^{\circ} \mathrm{C}$. For TEM measurement, the samples were dropped onto a copper net and the morphologies of liposomes were recorded with JEOL JEM-1400.

\section{Membrane fluidity of liposome}

The membrane fluidity of liposome was determined at $25{ }^{\circ} \mathrm{C}$ by a fluorophotometer (Perkin Elmer, LS55). $1.864 \mathrm{~mL}$ of $1.0 \mathrm{mg} / \mathrm{mL}$ liposome suspension was incubated with $2.5 \mathrm{~mL}$ of $2 \mu \mathrm{M} \mathrm{DPH}$ in water and then diluted to $5 \mathrm{~mL}$ at $25^{\circ} \mathrm{C}$ for $60 \mathrm{~min}$. Then the fluorescence (FL) intensity of DPH was measured at excitation and emission wavelengths of 360 and $425 \mathrm{~nm}$, respectively. The values of FL polarization $(P)$ were calculated using an analysis software (FL Winlab, Perkin Elmer, Co.) from the fluorescent intensity of 1,6-phenyl-1, 3, 5-hexatriene (DPH, Sigma) according to Eq. (1) (Kobayashi et al. 2007); the reciprocal value of polarization $(1 / P)$ was defined as the membrane fluidity: 


$$
P=\left(I_{V V}-G I_{V H}\right) /\left(I_{V V}+G I_{V H}\right)
$$

where $I_{\mathrm{VV}}$ was the FL intensity measured with both excitation and emission polarized vertically, and $I_{\mathrm{VH}}$ with the vertically polarized excitation and horizontally polarized emission. The $G$-factor $(G)$ was determined by the equipment as an impact factor, which is equal to $I_{\mathrm{HV}} / I_{\mathrm{HH}}$.

\section{Load and release of fluorescein}

Liposomes were prepared as mentioned above except that the lipid membrane was hydrated with a fluorescein $(400 \mu \mathrm{M})$ contained Tris- $\mathrm{HCl}$ buffer solution $(10 \mathrm{mM}$, $\mathrm{pH}$ 7.4). The not-incorporated fluorescein molecules were removed by gel permeation chromatography on a Sephadex G50 resin column, which was preconditioned with PBS (pH 7.4). The fluorescein left in gel column was measured for calculating the loading amount in gel layer. The weight ratio of incorporated fluorescein to lipid was calculated to be $1 / 100(\mathrm{~g} / \mathrm{g}) .2 \mathrm{~mL}$ of fluorescein loaded liposome was sealed in a dialysis tube (MWCO 3500), which was placed in $30 \mathrm{~mL}$ of phosphate buffered saline (PBS, pH 7.4). The fluorescein leakages were investigated at $37^{\circ} \mathrm{C}$ by using a spectrfluorometer (F-4500, Hitachi, Japan). The emission and excitation wavelengths were $517 \mathrm{~nm}$ and $495 \mathrm{~nm}$. Complete release of fluorescein was obtained from controlling ethanol-treated liposome. The accumulated leakage percentage was then determined by the FL intensity relative to $100 \%$ dequenching.

\section{Preparation of plasmid DNA}

Plasmid DNA pIRES2-EGFP (pDNA) was purchased from BD Biosciences Clontech, USA. EndoFree Plasmid Maxi Kit (Qiagen, Germany) was used to prepare high-quality endotoxin free plasmid. Briefly, pDNA was transferred into competent cell Escherichia coli DH $5 \alpha$ and streaked on kanamycin plate. A single colony was picked from the freshly selective plate and inoculated a starter culture of $3 \mathrm{~mL}$ Luria-Bertani medium containing $50 \mu \mathrm{g} / \mathrm{mL}$ kanamycin for $6 \mathrm{~h}$ at $37^{\circ} \mathrm{C}$ with $300 \mathrm{rpm}$ shaking. The starter culture was diluted at $1 / 500$ into $200 \mathrm{~mL}$ LB medium containing $50 \mu \mathrm{g} / \mathrm{mL}$ kanamycin, and grew at $37^{\circ} \mathrm{C}$ for $12 \mathrm{~h}$ with vigorous shaking $(300 \mathrm{rpm})$. After inoculation, the bacterial cells were harvested by centrifugation at $4000 \times g$ for $15 \mathrm{~min}$ at $4{ }^{\circ} \mathrm{C}$. After being re-suspended, lyses and neutralization, the pDNA was released from the lysate. Being recovered and purified with QIAGEN-tips, the pDNA was eventually resolved in $500 \mu \mathrm{L}$ endotoxin-free Buffer TE at a final concentration of $1.2 \mu \mathrm{g} / \mu \mathrm{L}$.

\section{Evaluation of pDNA condensation efficiency of liposome}

pDNA was diluted with Tris-HCl buffer ( $\mathrm{pH}$ 7.4) and mixed with liposome suspension at a terminal final concentration of $3.6 \mu \mathrm{g} / \mathrm{mL}$. The mixture was kept for $30 \mathrm{~min}$ at room temperature before further experiment. The amount of pDNA protected from intercalation of $\mathrm{EtBr}$ by liposome was evaluated by ethidium intercalation assay $(\mathrm{EtBr}$ was excited at $520 \mathrm{~nm}$ to produce a FL emission at $595 \mathrm{~nm}$ ). The pDNA composition efficiency was obtained through dividing the relative FL intensity of EtBr added to pDNA encapsulated in liposome by the maximum FL intensity of which added to free pDNA. 


\section{In vitro cellular internalization effect of liposome to Huh7 cells}

Rhodamine 6G loaded liposomes were prepared as mentioned above. Hepatocarcinoma cell line Huh7 was recovered from liquid nitrogen using DMEM (Gbico, USA) culture media with $10 \%$ FBS and P-S antibiotics, and passed 2-3 generations before use. Cells were placed in a volume of $500 \mu \mathrm{L}$ growth medium without antibiotics per well in a 24-well plate 1 day before transfection. The cells would be $90-95 \%$ confluent at the time of transfection. The liposome suspension $(30 \mu \mathrm{g} / \mathrm{mL}, 200 \mu \mathrm{L} /$ per well $)$ was transfected to 24-well plate containing 90-95\% confluent fresh cells and was rinsed by growth medium after 15 or 30 min incubation individually. Then the samples were imaged under a FL microscopy.

\section{Results}

\section{Characterization of galacto-liposomes}

Isomerism has been suggested to impact the LB monolayer property of glycolipids as well as the cell uptake of glycopolymer-containing nanoparticles (Liu et al. 2015). We have recently determined that anomerism, which is a common identity of nearly all glycoconjugates, could influence largely the bioactivity of glycolipids (He et al. 2011). Despite the extensive efforts in the construction of glyco-liposomes, investigations as regards the impact of anomerism on glyco-liposomes have been elusive.

Here we used a pair of anomeric galactolipids with a 16-carbon lipid chain, synthesized in a previous study (He et al. 2011), to test their liposomal properties while imbedded in a phospholipid-based liposome (Fig. 2). Liposomes that consist of pure DPPE (LipoDPPE), DPPE embedded with $\alpha$-galactolipid 1 (Lipo1) or DPPE embedded with $\beta$-galactolipid 2 (Lipo2) of a similar particle size were fabricated. The mixing ratio was below $30 \%$ as a previous study has suggested (Ueno et al. 2007) that further over-mixing would compromise the stability of glyco-liposomes.

The sizes and the Zeta-potentials of pure DPPE and galactolipid-incorporated liposomes were measured. The galacto-liposomes containing 10,20 or $30 \%$ of galactolipids were used for investigation. Their average sizes were determined to be $150 \pm 10 \mathrm{~nm}$ (PDI: 0.15) after filtration by an extruder equipped with a polycarbonate membrane (pore diameter: $200 \mathrm{~nm}$ ). Figure 3 shows the typical TEM images of the liposomes, indicating the doping of galactolipid did not give any influence on shape of liposomes. Their

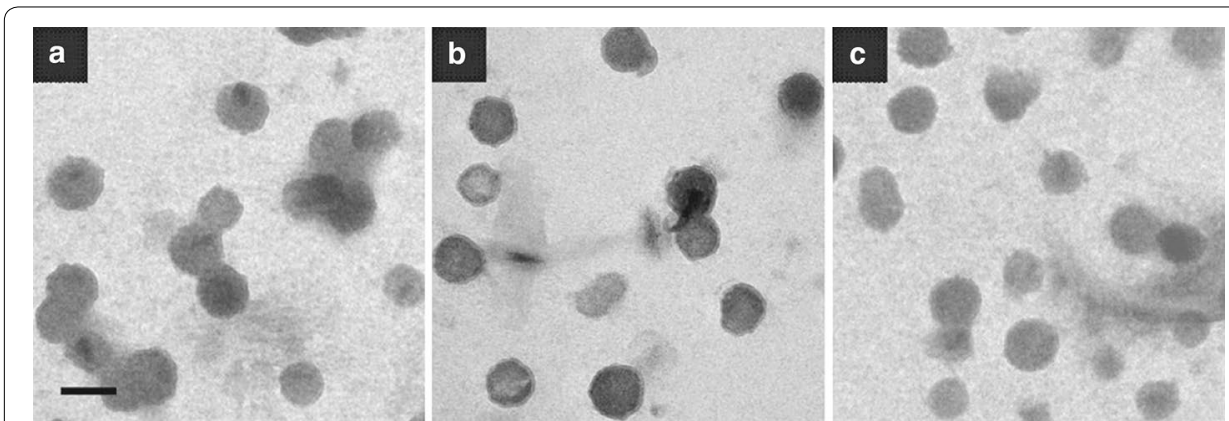

Fig. 3 Typical scanning electron microscope image of a LipoDPPE, b Lipo1 and c Lipo2 (20\% galactolipid fraction). Bar $200 \mathrm{~nm}$ 
Zeta-potential was identically $45 \pm 2 \mathrm{mV}$ irrespective of the glycolipid doping ratio. The plenty of charge give the liposome suspension better stability to avoid the assembly.

The phase transition temperature $\left(T_{\mathrm{m}}\right)$ of these liposomes were measured by using a DSC to test their thermo-stability. The typical DSC data shown in Fig. 4 suggests different decreases in $T_{\mathrm{m}}$ after imbedding the anomeric galactolipids into the DPPE liposome. Figure 5 a shows a complete $T_{\mathrm{m}}$ comparison among the liposomes. The $T_{\mathrm{m}}$ value of LipoDPPE was measured to be $67^{\circ} \mathrm{C}$, which is similar to a literature report $\left(64{ }^{\circ} \mathrm{C}\right.$ ) (Sun et al. 2014). In contrast, it decreased gradually with the increase of the doping ratio of galactolipids. The $T_{\mathrm{m}}$ values decreased to 60 and $52{ }^{\circ} \mathrm{C}$ for Lipo1 and Lipo2, respectively, which suggests that the thermal stability decreases when the galactolipids are present.

The membrane fluidity of liposomes is an important parameter, which can influence liposomal biofunctions such as drug encapsulation and drug release. Indeed, higher membrane fluidity can result in a quicker drug leakage or releasing. The $1 / P$ values, which are proportional to the membrane fluidity of the liposome, are calculated from the DPH FL polarizations (Fig. 5b) (Treichel et al. 1994). We observed that the $1 / P$ values of LipoDPPE increased with increasing galactolipid, while the 1/P value of Lipo1 was lower than that of Lipo2.

\section{The drug leakage of liposomes}

The stability of liposome could also be evaluated by the accumulated leakage of incorporated FL from various liposomes. The dynamics curves of FL release were obtained by measuring the FL intensity at regular intervals (Fig. 6). As expected, it showed an increase tendency with increasing molar ratio of galactolipid, agreeing with the results
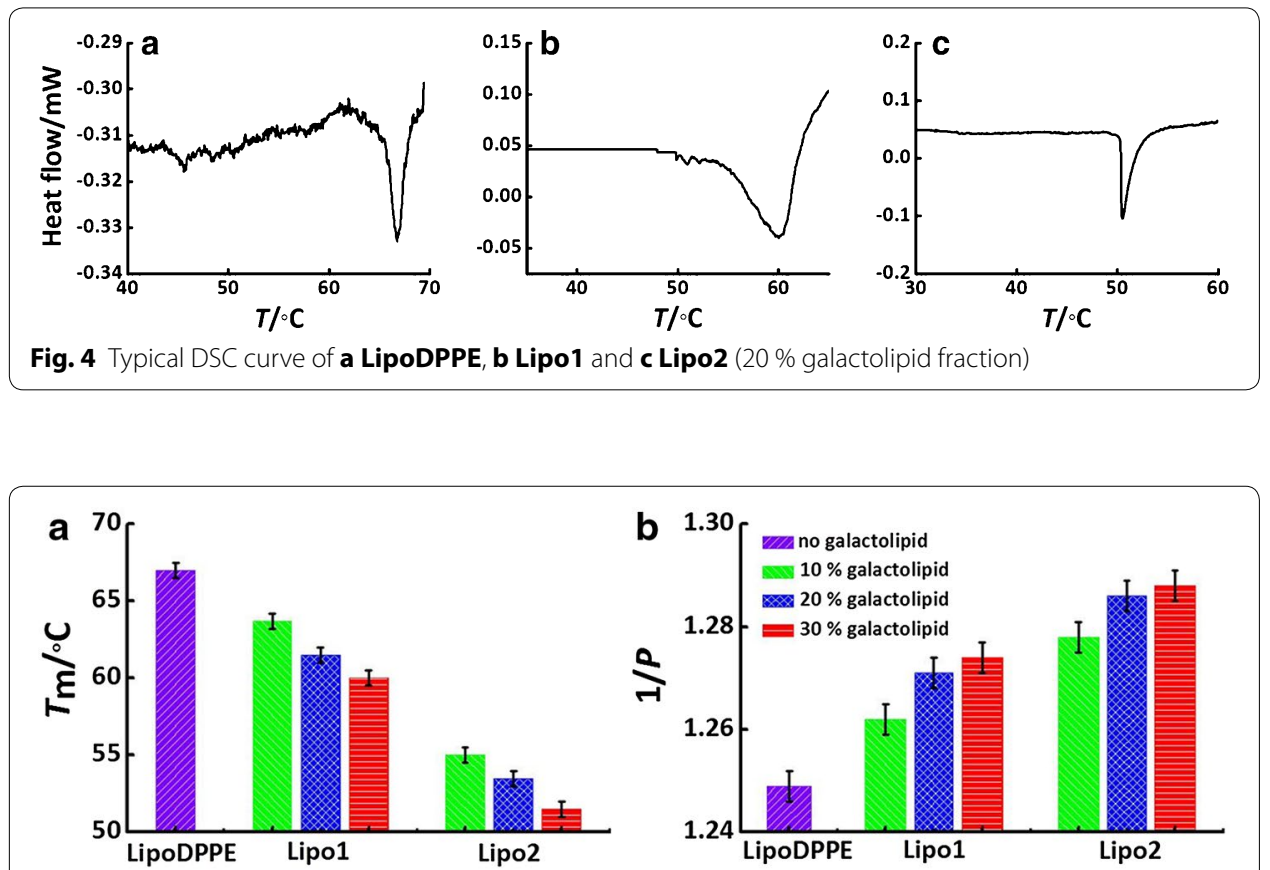

Fig. 5 a $T_{m}$ depicting the thermal stability $\left(T_{m}\right.$ of pure DPPE has been reported to be $64^{\circ} \mathrm{C}$ ) (Seddon et al. 1983), b 1/P values depicting the fluidity of LipoDPPE, Lipo1 and Lipo2 (10, 20 or $30 \%$ galactolipid fraction) 


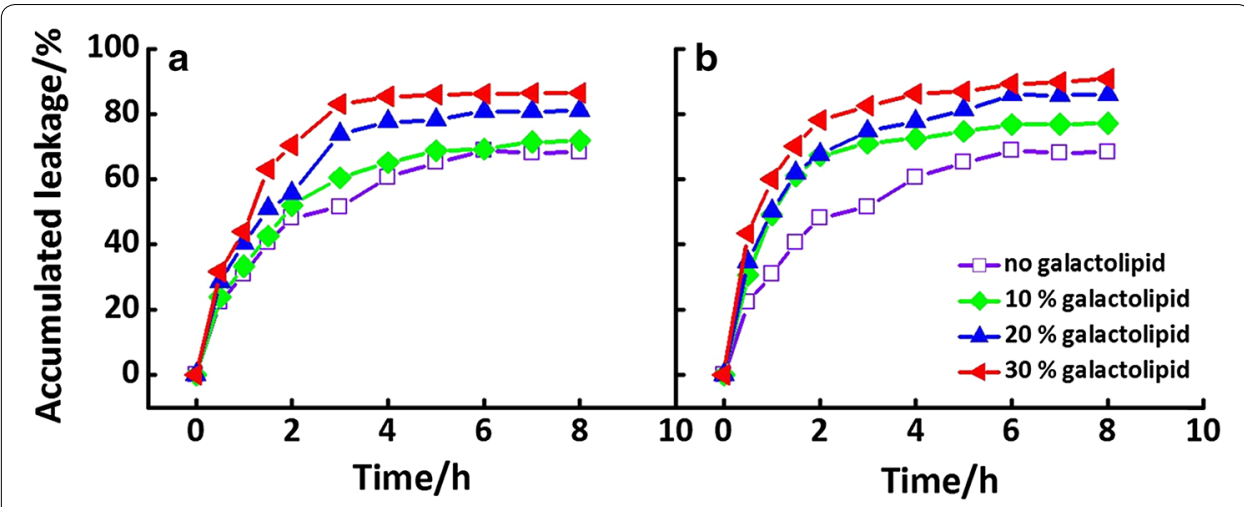

Fig. 6 Dynamics curves of fluorescence leakage from galacto-liposomes, Lipo1 (a) and Lipo2 (b). The results of pure liposome were also shown in both

of $1 / P$, as shown in Fig. $5 \mathrm{~b}$. The accumulated leakages of Lipo2 were slightly higher than those of Lipo1, indicating less stable membrane of the former.

\section{DNA condensation efficiency}

The DNA condensation efficiencies of the pure liposome and galacto-liposome were judged through the FL intensity of EtBr. When DNA molecules were encapsulated or condensed, EtBr cannot insert into DNA base pairs and then fluoresce. A plasmid DNA that encodes the internal ribosome entry site and the enhanced green fluorescent protein (pIRES2-EGFP) was employed.

Primarily, the size and Zeta-potential of the DNA-liposome complex were measured. Unlike the liposomes without DNA, the sizes of galacto-liposome increased a lot. The sizes of Lipo1 (3000-3500 nm) increased more than those of Lipo2 (1200-1500 nm), suggesting the attachment of DNA to liposome and, probably, formation of some aggregations. We observed that the efficiency of the anomeric galacto-liposomes was better than the pure DPPE liposome, whereas that of Lipo1 superior to Lipo2 (Fig. 7). And, the results also showed the DNA condensation changed a little with galactolipid ratios.

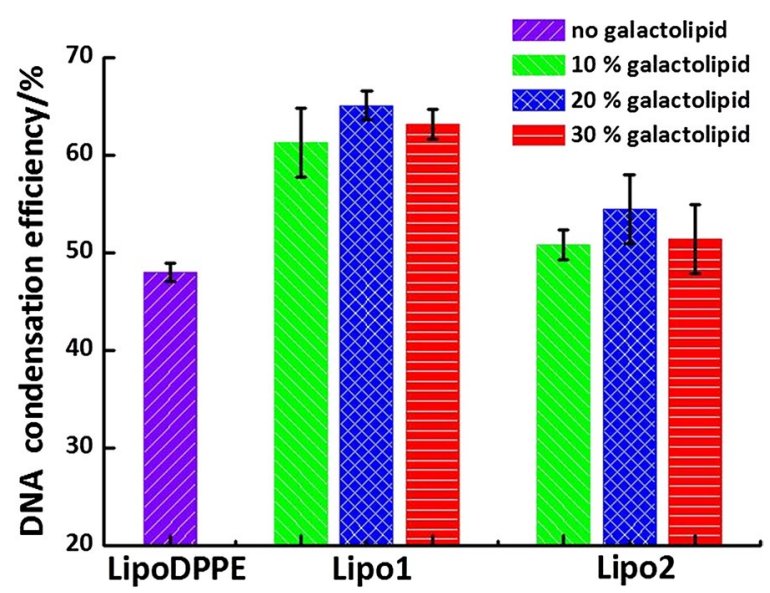

Fig. 7 DNA condensation efficiency of LipoDPPE, Lipo1 and Lipo2 
Their variation trend was not like the results of $T_{\mathrm{m}}$ and $1 / P$, but a slightly maximum appeared at the doping ratio of $20 \%$.

\section{In vitro cellular internalization}

The degree of cellular internalization of liposomes is a key parameter when used as drug/ gene carrier. Here, the cellular internalization of these liposomes (with or without $10 \%$ of galactolipid) towards a hepatoma cell line, Huh 7 that expresses ASGP-R, was evaluated. Cells were incubated with the liposomes for 15 or $30 \mathrm{~min}$ and then the FL was imaged (Fig. 8). Both anomeric galacto-liposomes (Fig. 8, Lipo1 and Lipo2) were observed to show somewhat stronger FL than the pure DPPE liposome (Fig. 8 LipoDPPE). Comparing the two galactolipid-liposomes, Lipo1 containing $\alpha$-galactolipid showed no obvious difference from Lipo2 containing $\beta$-galactolipid judged from the FL images. Then a quantitative results of liposomes embedded with various ratios of galactolipid were shown in Fig. 9 with LipoDPPE used as control.

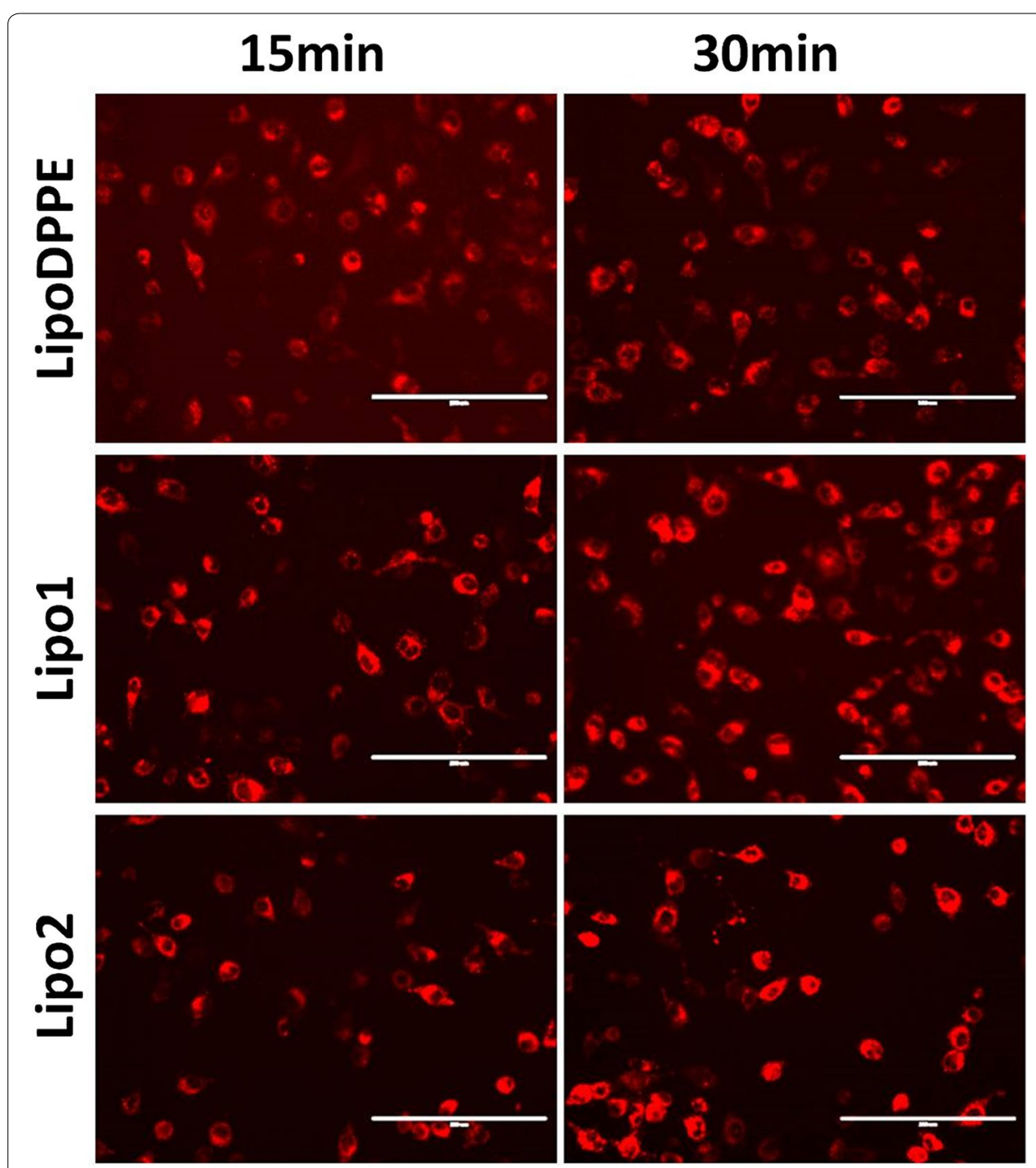

Fig. 8 Fluorescence imaging of Huh7 cells (scale bar $200 \mu$ M) after transfection by Lipo DPPE, Lipo1, Lipo2. Culturing time: left $15 \mathrm{~min}$, right $30 \mathrm{~min}$ (10\% galactolipid fraction) 


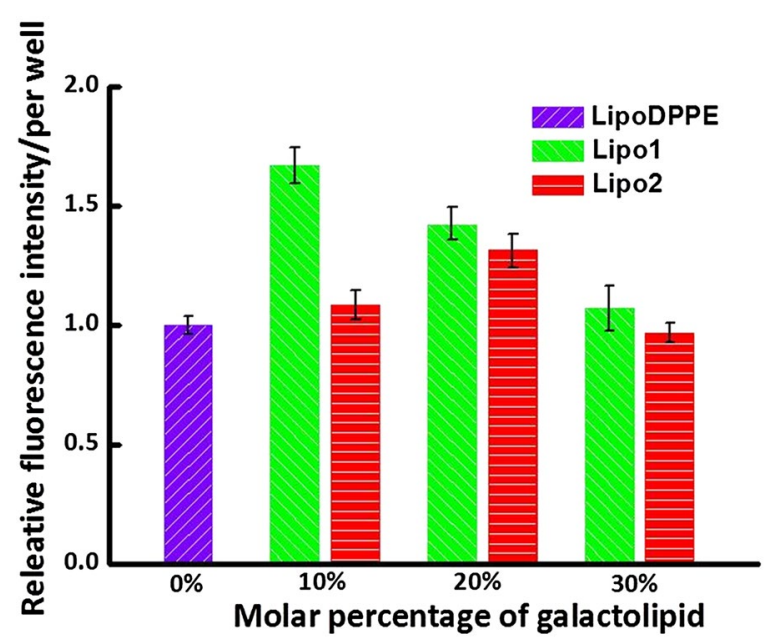

Fig. 9 Releative fluorescence intensity of per well after 30 min incubation with LipoDPPE, Lipo1 and Lipo2

\section{Discussions}

As galactolipid was incorporated into liposomes, the influence of the galactolipid on liposomes was characteries by DSC, FL polarization and drug leakage experiment. As shown in Fig. 5a, obviously, the imbedding of $\mathbf{2}$ has a larger impact on the stability of the liposomal property than $\mathbf{1}$. It is known that imbedding lipids with unsaturated carbon bond or with shorter carbon chain should decrease $T_{\mathrm{m}}$ values and then thermo-stability. Here, being imbedded into the DPPE liposome, the anomeric galactolipids could probably have different interactions with DPPE which results from their small configurational difference as mentioned in a previous paper (Liu et al. 2015). The observation that the $T_{\mathrm{m}}$ of Lipo1 is higher than Lipo2 (Fig. 5a) might be caused by a stronger intermolecular interaction of the $\alpha$-galacto-liposome than the $\beta$-counterpart, leading to the increased thermal stability.

From Fig. 5b, the $1 / P$ values of LipoDPPE were found to increase with content of galactolipid, suggesting that the DPPE molecules are disturbed by addition of the galactolipid molecules. And Lipo1 had lower 1/P value than Lipo2, which is also thought to be related to the interaction between DPPE and the anomeric galactolipids. A stronger interacton might hamper the motion of the moleclues in the bilayer.

For the fluorescein leakage experiment shown in Fig. 5b, their accumulated leakages increased with content of galactolipid and $1 / P$ value. The accumulated leakages of Lipo2 were slightly higher than those of Lipo1, indicating the less stable membrane of the former. As a drug carrier, high drug leakage is thought to increase side-effect of drug during cycling in body. However, too stable liposome might have another problem, that is, might tend to hardly release even if the carrier gets to the sick site. So it is necessary to study on drug release system which could release smartly and controllably.

From these results, we observed that, with the increase of the molar fraction of galactolipids, the thermal stability decreased and the fluidity of the liposome membrane increased. The higher membrane fluidity may account for its lower thermal stability. This is because the two properties might both relate directly to the motion of lipid carbon chain. 
The ability of these liposomes to condense DNA was also investigated. Although it has been suggested that the charge amount, fluidity of membrane and alkyl chain length of lipsome had impact on the DNA condensation and transfection efficiency, the exact mechanisms are not known (Lentz 1993). It is easy to think that the DNA condensation efficiency should firstly depend on the Zeta-potential and size of the liposome, which have been unified in this experiment. And secondly, the micro-property of liposomal membrane, including membrane fluidity, intermolecular interaction, molecular density, etc., should be concerned. According to the discussion mentioned above, we focus on the first two. The difference in DNA condensation between Lipo1 and Lipo2 could probably be ascribed to the slightly higher fluidity and weaker molecular interaction of the $\beta$ - than the $\alpha$-galacto-liposome (Song et al. 2012), which is unfavourable to condense the DNA strand more efficiently. We deduced that the liposomal membrane with higher fluidity (Lipo2) might hamper the process of DNA adsorption and condensation, leading to decreased DNA condensation efficiency.

As for the cellular internalization ability of these liposomes, within the short incubation with cells, there was very little amount of rhodamine released from liposomes. So it could be thought that there was very little free rhodamine could enter into cells through permeabilization. The intensity of FL was used to judge the amount of internalized liposomes. It was found that the difference between the results of Lipo1 and Lipo2 was not significant from the FL images. However, both anomeric galacto-liposomes showed better internalization ability than the pure DPPE liposome, suggesting that the presence of the galactoside warhead might give an improvement in the function of cellular internalization.

From the quantitative results of internalization, it was found that increase the ratio of galactolipid did not improve the internalization linearly, suggesting this bioprocess of cellular internalization should be very complex. But we could confirm that it was impacted seriously by membrane properties. An appropriate fluidity of liposomal membrane should be necessary. It was indicated a lower containing ratio of galactolipid seemed to get a less drug leakage and a better internalization effect.

We note that obvious difference was observed between the anomeric counterparts (Lipo1 and Lipo2), suggesting that further sophisticated experiments are needed to elaborate such difference. However, the above-shown cellular data clearly demonstrated the advantage of using Lipo1 for preparing hepatoma cell targeted drug carriers.

\section{Conclusions}

As what discussed above, using a pair of anomeric galactolipids we showed that their liposomal properties and biofunctions, while incorporated with a DPPE based liposome, were different as a result of their small configurational difference between $\alpha$ - and $\beta$-galacolipid. Galacto-liposomes (Lipo1 and Lipo2) showed decreased thermal stability and increased membrnane fluidity, leading to the better DNA condensation efficiency probably due to stronger ability to bind and condense DNA chains. And also the all galacto-liposomes, especially Lipo1, showed better internalization ability than the pure DPPE liposome. We suggest that the elaboration of the structural and functional distinction between isomeric glyco-liposomes should be an important task to address in the field of targeted drug delivery, shedding light on the better understanding of the endocytosis and membrane fusion of these vesicles on the cellular level. 


\section{Authors' contributions}

The manuscript was conceived and designed by XSH and LHL. LDY and ZJQ performed acquisition and analysis of data. All authors were involved in revising the manuscript. All authors read and approved the final manuscript.

\section{Author details}

'Key Laboratory for Advanced Materials and Institute of Fine Chemicals, School of Chemistry and Molecular Engineering, East China University of Science and Technology, 130 Meilong Rd, Shanghai 200237, People's Republic of China.

${ }^{2}$ Key Laboratory of Medical Molecular Virology (Ministry of Health and Ministry of Education), School of Basic Medical Sciences, Fudan University, 138 Yixueyuan Rd, Shanghai 200032, People's Republic of China.

\section{Acknowledgements}

The galactolipid was kindly provided by Prof. Xiao-Peng He. This work is supported by the National Natural Science Foundation of China $(21276074,91334203)$ and the Fundamental Research Funds for the Central Universities.

\section{Competing interests}

The authors declare that they have no competing interests.

Received: 13 January 2016 Accepted: 26 April 2016

Published online: 17 May 2016

\section{References}

Bertozzi CR, Kiessling LL (2001) Chemical glycobiology. Science 291(5512):2357-2364

Hart GW, Copeland RJ (2010) Glycomics hits the big time. Cell 143(5):672-676

He X-P, Xu X, Zhang H-L, Chen G-R, Xu S, Liu H (2011) Disclosing the distinct interfacial behaviors of structurally and configurationally diverse triazologlycolipids. Carbohydr Res 346(11):1320-1326

Hu Y, Haynes MT, Wang Y, Liu F, Huang L (2013) A highly efficient synthetic vector: nonhydrodynamic delivery of DNA to hepatocyte nuclei in vivo. ACS Nano 7(6):5376-5384

Jain K, Kesharwani P, Gupta U, Jain NK (2012) A review of glycosylated carriers for drug delivery. Biomaterials 33(16):4166-4186

Jayaraman N, Maiti K, Naresh K (2013) Multivalent glycoliposomes and micelles to study carbohydrate-protein and carbohydrate-carbohydrate interactions. Chem Soc Rev 42(11):4640-4656

Kobayashi T, Ishida T, Okada Y, Ise S, Harashima H, Kiwada H (2007) Effect of transferrin receptor-targeted liposomal doxorubicin in P-glycoprotein-mediated drug resistant tumor cells. Int J Pharm 329(1-2):94-102

Lentz BR (1993) Use of fluorescent probes to monitor molecular order and motions within liposome bilayers. Chem Phys Lipids 64(1):99-116

Liu D, Wang W, He X, Chen G, Xu S, Liu H (2015) Anomerism differentiates the interfacial property of galactolipid-doped phospholipid monolayers. Issues Biol Sci Pharm Res 3(8):78-85

Marth JD, Grewal PK (2008) Mammalian glycosylation in immunity. Nat Rev Immunol 8(11):874-887

Rädler JO, Koltover I, Salditt T, Safinya CR (1997) Structure of DNA-cationic liposome complexes: DNA intercalation in multilamellar membranes in distinct interhelical packing regimes. Science 275(5301):810-814

Ramezani M, Khoshhamdam M, Dehshahri A, Malaekeh-Nikouei B (2009) The influence of size, lipid composition and bilayer fluidity of cationic liposomes on the transfection efficiency of nanolipoplexes. Colloids Surf B 72(1):1-5

Rigopoulou El, Roggenbuck D, Smyk DS, Liaskos C, Mytilinaiou MG, Feist E, Conrad K, Bogdanos DP (2012) Asialoglycoprotein receptor (ASGPR) as target autoantigen in liver autoimmunity: lost and found. Autoimmun Rev 12(2):260-269

Róg T, Vattulainen I, Bunker A, Karttunen M (2007) Glycolipid membranes through atomistic simulations: effect of glucose and galactose head groups on lipid bilayer properties. J Phys Chem B 111(34):10146-10154

Seddon JM, Cevc G, Marsh D (1983) Calorimetric studies of the gel-fluid (L.beta.-L.alpha.) and lamellar-inverted hexagonal (L.alpha.-HII) phase transitions in dialkyl- and diacylphosphatidylethanolamines. Biochemistry 22(5):1280-1289

Seeberger PH (2005) Exploring life's sweet spot. Nature 437(7063):1239

Sliedregt LAJM, Rensen PCN, Rump ET, van Santbrink PJ, Bijsterbosch MK, Valentijn ARPM, van der Marel GA, van Boom $J H$, van Berkel TJC, Biessen EAL (1999) Design and synthesis of novel amphiphilic dendritic galactosides for selective targeting of liposomes to the hepatic asialoglycoprotein receptor. J Med Chem 42(4):609-618

Song S-X, Wu M-L, He X-P, Zhou Y-B, Sheng L, Li J, Chen G-R (2012) The anomeric mixture of some O-galactolipid derivatives is more toxic against cancer cells than either anomer alone. Bioorg Med Chem Lett 22(5):2030-2032

Stimac A, Segota S, Dutour Sikiric M, Ribic R, Frkanec L, Svetlicic V, Tomic S, Vranesic B, Frkanec R (2012) Surface modified liposomes by mannosylated conjugates anchored via the adamantyl moiety in the lipid bilayer. Biochim Biophys Acta 1818(9):2252-2259

Sun P, He Y, Lin M, Zhao Y, Ding Y, Chen G, Jiang M (2014) Glyco-regioisomerism effect on lectin-binding and cell-uptake pathway of glycopolymer-containing nanoparticles. ACS Macro Lett 3(1):96-101

Symens N, Méndez-Ardoy A, Díaz-Moscoso A, Sánchez-Fernández E, Remaut K, Demeester J, Fernández JMG, De Smedt SC, Rejman J (2012) Efficient transfection of hepatocytes mediated by mRNA complexed to galactosylated cyclodextrins. Bioconjug Chem 23(6):1276-1289

Treichel U, Meyer zum Büschenfelde K-H, Stockert RJ, Poralla T, Gerken G (1994) The asialoglycoprotein receptor mediates hepatic binding and uptake of natural hepatitis B virus particles derived from viraemic carriers. J Gen Virol 75(11):3021-3029

Ueno Y, Hirashima N, Inoh Y, Furuno T, Nakanishi M (2007) Characterization of biosurfactant-containing liposomes and their efficiency for gene transfection. Biol Pharm Bull 30(1):169-172

Varki A (1993) Biological roles of oligosaccharides: all of the theories are correct. Glycobiology 3(2):97-130 
Weis WI, Taylor ME, Drickamer K (1998) The C-type lectin superfamily in the immune system. Immunol Rev 163(1):19-34 Yang Y, Yang Y, Xie X, Cai X, Zhang H, Gong W, Wang Z, Mei X (2014) PEGylated liposomes with NGR ligand and heat-activable cell-penetrating peptide-doxorubicin conjugate for tumor-specific therapy. Biomaterials 35(14):4368-4381

Yin L, Song Z, Kim KH, Zheng N, Gabrielson NP, Cheng J (2013) Non-viral gene delivery via membrane-penetrating, mannose-targeting supramolecular self-assembled nanocomplexes. Adv Mater 25(22):3063-3070

Zhao Q, Chen W, Chen Y, Zhang L, Zhang J, Zhang Z (2011) Self-assembled virus-like particles from rotavirus structural protein VP6 for targeted drug delivery. Bioconjug Chem 22(3):346-352

\section{Submit your manuscript to a SpringerOpen ${ }^{\circ}$} journal and benefit from:

- Convenient online submission

- Rigorous peer review

- Immediate publication on acceptance

- Open access: articles freely available online

- High visibility within the field

- Retaining the copyright to your article

Submit your next manuscript at $\boldsymbol{\nabla}$ springeropen.com 